\title{
PU.1 serves a critical role in the innate defense against Aspergillus fumigatus via dendritic cell-associated C-type lectin receptor-1 and toll-like receptors-2 and 4 in THP-1-derived macrophages
}

\author{
CHENYANG LIU ${ }^{1,2 *}$, MIN WANG $^{3 *}$, WENKUI SUN $^{2}$, FENG CAI $^{4}$, SHEN GENG $^{1}, \mathrm{XIN} \mathrm{SU}^{2}$ and YI SHI ${ }^{1,2}$ \\ ${ }^{1}$ Graduate School, Southern Medical University, Guangzhou, Guangdong 510515; ${ }^{2}$ Department of \\ Respiratory and Critical Care Medicine, Jinling Hospital Affiliated to Southern Medical University, \\ Nanjing, Jiangsu 210002; ${ }^{3}$ Department of Respiratory Medicine, Shanghai First People's Hospital, \\ Shanghai 200080; ${ }^{4}$ Medical School of Nanjing University, Nanjing, Jiangsu 210093, P.R. China
}

Received February 20, 2016; Accepted February 10, 2017

DOI: $10.3892 / \mathrm{mmr} .2017 .6504$

\begin{abstract}
Aspergillus fumigatus (A. fumigatus) is one of the most common fungal pathogens of invasive pulmonary aspergillosis (IPA), which may be life threatening in immunocompromised individuals. The dendritic cell-associated C-type lectin receptor (Dectin-1), toll-like receptor (TLR)-2 and TLR-4 are major pattern recognition receptors in alveolar macrophages that recognize A. fumigatus components. The PU.1 transcription factor is known to be important for the transcriptional control of these three receptors in mature macrophages. The present study investigated whether alterations of PU.1 expression may affect the innate defense against $A$. fumigatus in the human monocyte THP-1 cell line. THP-1-derived macrophages were transduced with PU.1 adenoviral vectors and transfected with PU.1 small interfering RNA, and the mRNA and protein expression levels of Dectin-1, TLR-2 and TLR-4 were measured. In addition, the levels of tumor necrosis factor (TNF)- $\alpha$ and interleukin (IL)-1 $\beta$ were ascertained, and fungal phagocytosis and killing were assessed. The results demonstrated that overexpression of PU.1 by recombinant adenoviral vectors resulted in a significant upregulation of Dectin-1, TLR-2 and TLR-4 at
\end{abstract}

Correspondence to: Professor Yi Shi or Professor Xin Su, Department of Respiratory and Critical Care Medicine, Jinling Hospital Affiliated to Southern Medical University, 305 Zhongshan East Road, Nanjing, Jiangsu 210002, P.R. China

E-mail: shiyi56@126.com

E-mail: suxinjs@163.com

*Contributed equally

Key words: PU.1, dendritic cell-associated C-type lectin receptor-1, toll-like receptor-2, toll-like receptor-4, Aspergillus fumigatus, innate immunity the transcriptional and translational levels. In response to A. fumigatus stimulation, PU.1 overexpression increased TNF- $\alpha$ and IL-1 $\beta$ production. In addition, Dectin-1, TLR-2 and TLR-4 upregulation may have enhanced the phagocytosis and killing ability of THP-1-derived macrophages. As expected, silencing of PU.1 led to downregulation of Dectin-1, TLR-2, TLR-4 and the expression of pro-inflammatory cytokines, as well as decreased phagocytosis and the killing ability of THP1-derived macrophages. In conclusion, the results indicate that PU.1 may be a critical factor for the innate defense against A. fumigatus, and may therefore be a potential target for the prophylaxis and treatment of IPA.

\section{Introduction}

Aspergillus fumigatus (A. fumigatus) is one of the most important opportunistic pathogens that form airborne spores (conidia) in our environment (1). Immunocompromised individuals may develop invasive aspergillosis (IA), which demonstrates high morbidity and mortality rates (2). Invasive pulmonary aspergillosis (IPA) is the most common form of IA, and affects immunocompromised hosts including those with malignancies, advanced human immunodeficiency virus infection, patients that have undergone hematopoietic stem cell transplantation and those receiving immunosuppressive treatments (3). Due to the increasing number of immunocompromised patients, the number of cases of IPA is increasing substantially (3). Thus, an improved understanding of the host defense against $A$. fumigatus is urgently required.

Alveolar macrophages (AMs) serve a critical role in the host's innate defense against $A$. fumigatus. AMs express several different pattern recognition receptors (PRRs) that mediate recognition of the invading pathogens via pathogen-associated molecular patterns (4). The A. fumigatus cell wall is mainly composed of chitin, $\alpha$-glucans, $\beta$ - $(1,3)(1,4)$-glucans and galactomannans, which are recognized by specific PRRs, including C-type lectin receptors (CTLs) and toll-like receptors (TLRs) (4). Dendritic cell-associated C-type lectin 
receptor (Dectin-1) is one of the most important CTLs that recognize the major fungal cell wall component, $\beta$-1,3-glucan. Dectin- $1^{-/-}$mice are more susceptible to A. fumigatus and produce fewer pro-inflammatory cytokines (5). Additionally, the presence of a Dectin-1 polymorphism increases the risk of IA in patients with allogeneic stem cell transplantation (6). In a recent study (3), overexpression of Dectin-1 in MH-S murine AM cells promoted cytokine release and killing ability during A.fumigatus stimulation. Among the TLRs, TLR-2 and TLR-4 have been extensively studied, and are key recognition components for host defense against A. fumigatus via the common TLR adaptor protein, myeloid differentiation primary response gene 88 (MyD88) (7). AMs from TLR-2-deficient mice produce fewer inflammatory cytokines in response to A. fumigatus, including tumor necrosis factor (TNF)- $\alpha$, chemokine (C-X-C motif) ligand 2 and macrophage inflammatory protein-2 $(8,9)$. TLR $-4^{-/}$mice and TLR-4 polymorphisms in donor cells from hematopoietic stem cell transplant recipients demonstrate an increased susceptibility to A. fumigatus $(10,11)$. Therefore, Dectin-1-dependent and MyD88-dependent pathways are critical for the host defense against $A$. fumigatus in mice and humans.

Human transcription factor PU.1 (encoded by the SPII gene) is a member of the E26-transformation-specific (Ets) family, which is important for macrophage development and the transcriptional control of inducible genes in mature macrophages (12). In mice and humans, PU.1 binds to the gene promoter and enhances the transcription of Dectin-1 $(13,14)$, TLR-2 $(15,16)$ and TLR-4 $(15,17,18)$. Based on the evidence that PU.1 may be critical for the regulation of these three receptors in macrophages, the authors of the present study hypothesized that alterations in the expression of PU.1 may affect the innate defense against $A$. fumigatus. In the present study, phorbol-12-myristate-13-acetate (PMA)-induced THP-1 derived macrophages were used as a model of AMs. The aim was to investigate whether alterations in the expression of PU.1 affects innate immune functions in AMs during A. fumigatus stimulation, including cytokine release, phagocytosis and the killing ability.

\section{Materials and methods}

THP-1 derived macrophages and preparation of conidia. The human monocyte cell line, THP-1 (American Type Culture Collection, Manassas, VA, USA) was cultured in RPMI 1640 (Gibco; Thermo Fisher Scientific, Inc., Waltham, MA, USA) containing 10\% FBS (Gibco; Thermo Fisher Scientific, Inc.) and $1 \%$ antibiotic-antimycotic solution (Gibco; Thermo Fisher Scientific, Inc.) at $37^{\circ} \mathrm{C}$ with $5 \% \mathrm{CO}_{2}$. To prepare THP-1-derived macrophages, THP-1 cells were incubated with PMA (cat. no. P1585; Sigma-Aldrich; Merck KGaA, Darmstadt, Germany) at a concentration of $100 \mathrm{ng} / \mathrm{ml}$ for $48 \mathrm{~h}$. Following incubation, adherent macrophages were maintained in complete medium at $37^{\circ} \mathrm{C}$ with $5 \% \mathrm{CO}_{2}$.

A.fumigatus $A 1$ was kindly provided by the Microbiological Laboratory of Jinling Hospital (Nanjing, China). A. fumigatus was cultured on Sabouraud dextrose agar (Thermo Fisher Scientific, Inc.) containing penicillin (100 U/ml) and streptomycin (100 $\mu \mathrm{g} / \mathrm{ml}$; Gibco; Thermo Fisher Scientific, Inc.) for 5 days at $37^{\circ} \mathrm{C}$.
Conidia were washed from agar plates using $5 \mathrm{ml}$ sterile phosphate-buffered saline (PBS) supplemented with $0.025 \%$ Tween-20. The suspension was filtered through nylon filters with $25-\mu \mathrm{m}$ pores to separate the conidia from the contaminating mycelium. Resting conidia were diluted in PBS and counted using a hemocytometer. To prepare fluorescein isothiocyanate (FITC)-labeled conidia, a total of $5 \times 10^{8} / \mathrm{ml}$ conidia were suspended in $0.1 \mathrm{M}$ carbonate buffer $(\mathrm{pH} 9.0)$ prior to incubation with FITC (cat. no. F-4274; Sigma-Aldrich; Merck $\mathrm{KGaA}$ ) at a final concentration of $0.16 \mathrm{mg} / \mathrm{ml}$ overnight at $4^{\circ} \mathrm{C}$. The suspension was then diluted and washed twice with PBS. FITC-labeled conidia were diluted in PBS to the desired concentration $\left(4 \times 10^{8} / \mathrm{ml}\right)$ and stored at $4^{\circ} \mathrm{C}$ in the dark $(3,19)$.

PU.1 gene overexpression. The PU.1 recombinant adenovirus vector used in the study was provided by Shanghai R\&S Biotechnology Co., Ltd. (Shanghai, China) and the final titer was $8 \times 10^{11} \mathrm{IU} / \mathrm{ml}$. THP-1 derived macrophages were seeded in six-well plates (Corning Incorporated, Corning, NY, USA) at a density of $8 \times 10^{5}$ cells/well. Cells were divided into the following three groups: Mock untransduced group; an empty-vector transduction group (Ad group), where cells were transduced at a multiplicity of infection (MOI) of 1,500; and a PU.1 recombinant adenovirus vector-transduced group (Ad-PU.1), where were transduced at an MOI of 1,500. Following co-culture in $1 \mathrm{ml}$ of RPMI 1640 medium for $12 \mathrm{~h}$, the adenovirus-containing medium was removed and replaced with complete RPMI 1640 medium. Total RNA and protein were extracted at $48 \mathrm{~h}$ post-transduction and stored at $-80^{\circ} \mathrm{C}$ until required.

PU.1 gene silencing. Small interfering RNA (siRNA) targeting PU.1 and negative control siRNA were obtained from Shanghai GenePharma Co., Ltd. (Shanghai, China). The sequence for the PU.1 siRNA was as follows: Forward, 5'-UAUAGAUCC GUGUCAUAGGGCACCA-3', and reverse, 5'-UGGUGCCCU AUGACACGGAUCUAUA-3'. THP-1-derived macrophages were seeded in six-well plates (Corning Incorporated) at a density of $8 \times 10^{5}$ cells/well. For each transfection, $160 \mathrm{pmol}$ PU.1 siRNA (siPU.1 group) or negative control siRNA (siNC group) was mixed with $8 \mu$ l Lipofectamine ${ }^{\circledR} 2000$ (Invitrogen; Thermo Fisher Scientific, Inc.) and $500 \mu$ l Opti-MEM (Invitrogen; Thermo Fisher Scientific, Inc.), and incubated at room temperature for $20 \mathrm{~min}$. The mixture was then added to each well containing the cells and the plate was incubated for $8 \mathrm{~h}$ at $37^{\circ} \mathrm{C}$. Cells that were untransfected were used as a mock transfection group. Following incubation, $1.5 \mathrm{ml}$ complete RPMI 1640 medium was added to each well. Total RNA and protein were extracted at $48 \mathrm{~h}$ post-transfection and stored at $-80^{\circ} \mathrm{C}$ until required.

Stimulation of cells with resting conidia. Following overexpression or silencing of PU.1, THP-1-derived macrophages were stimulated with $A$. fumigatus resting conidia (MOI=1, if not indicated otherwise) for $0,4,8$ and $12 \mathrm{~h}$, respectively. Total RNA and culture supernatants were then isolated at these indicated time points. Samples were stored at $-80^{\circ} \mathrm{C}$ until required.

Western blot analysis. Cells were lysed with radioimmunoprecipitation assay lysis buffer containing 
$25 \mathrm{mM}$ Tris- $\mathrm{HCl}$ (pH 7.6), $150 \mathrm{mM} \mathrm{NaCl}, 1 \% \mathrm{NP}-40$ and $1 \%$ sodium deoxycholate with $1 \%$ protease inhibitor cocktail (Sigma-Aldrich; Merck KGaA). The protein concentration was determined using a bicinchoninic acid assay protein assay kit (Beyotime Institute of Biotechnology, Hangzhou, China) according to the manufacturer's protocol. Protein lysates $(10 \mu \mathrm{g})$ were separated using 10\% SDS-polyacrylamide gels (cat. no. P0012A; Beyotime Institute of Biotechnology) and transferred to polyvinylidene difluoride membranes (GE Healthcare Life Sciences, Chalfont, UK). Membranes were then blocked with 5\% skimmed milk (Sigma-Aldrich; Merck $\mathrm{KGaA}$ ) for $2 \mathrm{~h}$ at room temperature. The membranes were subsequently hybridized with primary rabbit anti-human polyclonal antibodies against PU.1 (cat. no. 2258; Cell Signaling Technology, Inc., Danvers, MA, US), Dectin-1 (cat. no. 9051; Cell Signaling Technology, Inc.), TLR-2 (cat. no. 2229S; Cell Signaling Technology, Inc.) and TLR-4 (cat. no. sc-10741; Santa Cruz Biotechnology, Inc., Dallas, TX, USA) at 1:1,000 dilution overnight at $4{ }^{\circ} \mathrm{C}$. Membranes were also incubated with primary rabbit anti-human polyclonal antibodies against $\beta$-tubulin (cat. no. 2146S; Cell Signaling Technology, Inc.) as a loading control for PU.1, and $\beta$-actin (cat. no. 12620; Cell Signaling Technology, Inc.) for Dectin-1, TLR-2 and TLR-4, at a 1:1,000 dilution overnight at $4^{\circ} \mathrm{C}$. Following incubation with the relevant horseradish peroxidase-conjugated polyclonal goat anti-rabbit secondary antibody (cat. no. sc-2004; Santa Cruz Biotechnology, Inc.) at 1:5,000 dilution at room temperature for $2 \mathrm{~h}$, an enhanced chemiluminescence detection kit (Beyotime Institute of Biotechnology) was used for detection of immunoreactive proteins. Images of protein bands were acquired using the G:BOX Chemi XR5 system (Syngene, Frederick, MD, USA) (20). All antibodies were diluted in Tris-buffered saline with $0.1 \%$ Tween- 20 .

Reverse transcription-quantitative polymerase chain reaction $(R T-q P C R)$. Total RNA was isolated using TRIzol reagent (Invitrogen; Thermo Fisher Scientific, Inc.) according to the manufacturer's protocols. The concentration and purity of extracted RNA were (260/280 ratio) was determined using a NanoDrop ${ }^{\text {TM }} 2000$ spectrophotometer (Thermo Fisher Scientific, Inc.). cDNA was synthesized from total RNA $(1 \mu \mathrm{g})$ using PrimeScript RT Master Mix (cat. no. RR036A; Takara Biotechnology Co., Ltd., Dalian, China). The sequences of human-specific primers were synthesized by GenScript (Nanjing, China) and are listed in Table I. qPCR was performed using an Applied Biosystems Prism 7500 PCR system (Applied Biosystems; Thermo Fisher Scientific, Inc.) using SYBR ${ }^{\circledR}$ Premix Ex Taq ${ }^{\mathrm{TM}}$ (cat. no. RR420A; Takara Biotechnology Co., Ltd.). PCR was performed as follows: Denaturation at $95^{\circ} \mathrm{C}$ for $30 \mathrm{sec}$, followed by 40 cycles of $95^{\circ} \mathrm{C}$ for $5 \mathrm{sec}$ and $60^{\circ} \mathrm{C}$ for $30 \mathrm{sec}$. Relative mRNA expression levels were normalized to GAPDH, and calculated using the $2^{-\Delta \Delta \mathrm{Cq}}$ method (21).

Enzyme-linked immunosorbent assay (ELISA). TNF- $\alpha$ and interleukin (IL)-10 levels in the cell culture supernatants were determined using commercially available Quantikine ELISA kits (cat. nos. DTA00C and D1000B, respectively; R\&D Systems, Minneapolis, MN, USA), strictly according to the manufacturer's protocols. Absorbance was recorded at $450 \mathrm{~nm}$ in a microplate reader (Thermomax; Molecular Devices, LLC, Sunnyvale, CA, USA).

Confocal laser-scanning microscopy. THP-1-derived macrophages were seeded on $22 \times 22 \mathrm{~mm}$ coverslips in six-well plates (Corning Incorporated) at a density of $8 \times 10^{5}$ cells/well. Following transfection with PU.1 siRNA or transduction with Ad-PU.1 for $48 \mathrm{~h}$, cells were co-cultured with $4 \times 10^{6}$ FITC-labeled resting conidia $(\mathrm{MOI}=5)$ in $1 \mathrm{ml}$ complete RPMI 1640 medium at $37^{\circ} \mathrm{C}$ and $5 \% \mathrm{CO}_{2}$ for $4 \mathrm{~h}$. Cells were then washed vigorously three times with PBS, and fixed in $4 \%$ paraformaldehyde for $10 \mathrm{~min}$ at room temperature. The cells were gently washed three times with PBS and incubated with 1,1'-dioctadecyl-3,3,3',3'-tetramethylindocarbocyanine perchlorate (DiI; cat. no. C1036; dilution, 1:500; Beyotime Institute of Biotechnology) and DAPI (cat. no. C1002; dilution, 1:1,000; Beyotime Institute of Biotechnology) to stain the membrane and nucleus, respectively. Finally, the coverslips were removed, gently washed three times with PBS and mounted onto glass slides using SlowFade Gold antifade reagent (Invitrogen; Thermo Fisher Scientific, Inc.). A confocal laser-scanning microscope (Fluoview ${ }^{\circledR}$ FV10i; Olympus Corporation, Tokyo, Japan) with a x63 objective oil immersion lens was used to obtain all images. FITC-labeled resting conidia, cell membranes and nuclei were observed by the fluorescence intensities of FITC (green; excitation, 495 $\mathrm{nm}$; emission, $525 \mathrm{~nm}$ ), DiI (red; excitation, $549 \mathrm{~nm}$; emission range collected, $565 \mathrm{~nm}$ ) and DAPI (blue; excitation, $364 \mathrm{~nm}$; emission, $454 \mathrm{~nm})$.

Killing experiments. THP-1-derived macrophages $\left(8 \times 10^{5}\right.$ cells/well) were exposed to resting conidia $(\mathrm{MOI}=0.5)$ in six-well plates for $2 \mathrm{~h}$. Killing experiments were performed as previously described (3). Briefly, non-adherent cells and non-phagocytosed conidia were removed by washing the cells with pre-warmed PBS, and fresh medium was added ( $0 \mathrm{~h}$ time point). The cells were subsequently incubated for 4 or $8 \mathrm{~h}$. At 0,4 and $8 \mathrm{~h}$ time points, the plates were frozen at $-80^{\circ} \mathrm{C}$, and immediately thawed at $37^{\circ} \mathrm{C}$ to lyse the cells and harvest the conidia. Serial dilutions were performed in sterile medium, and solutions were used to inoculate Sabouraud dextrose agar (Thermo Fisher Scientific, Inc.). Colonies were counted following incubation for $24 \mathrm{~h}$ at $37^{\circ} \mathrm{C}$. The percentage of killed conidia was calculated by dividing the difference between the number of conidia recovered at 0 and 4 or $8 \mathrm{~h}$ by the total number of conidia recovered at $0 \mathrm{~h}$, and then multiplying by 100 (22).

Statistical analysis. Statistical analyses were performed using GraphPad Prism software (version 5.0; GraphPad Software, Inc., La Jolla, CA, USA) and the SPSS statistical analysis software program (version, 19.0; IBM SPSS, Armonk, NY, USA). All experiments were performed in triplicate. Data are presented as the mean \pm standard deviation. One-way analysis of variance followed by a Bonferroni post hoc test was used to compare differences among three groups when equal variances were assumed, and a Dunnett's T3 multiple comparisons test was used when equal variances were not assumed. $\mathrm{P}<0.05$ was considered to indicate a statistically significant difference. 
Table I. Primer sequences used for reverse transcription-quantitative polymerase chain reaction.

\begin{tabular}{lll}
\hline Gene & \multicolumn{1}{c}{ Forward primer $\left(5^{\prime}-3^{\prime}\right)$} & \multicolumn{1}{c}{ Reverse primer $\left(5^{\prime}-3^{\prime}\right)$} \\
\hline PU.1 & CGTGCACAGCGAGTTCGA & GCTCTGGAGCTCCGTGAAGT \\
Dectin-1 & GCAATACCAGGATAGCTGTTG & CCAAGCATAGGATTCCCAA \\
TLR-2 & CCTCTCGGTGTCGGAATGT & CATCCCGCTCACTGTAAGAAA \\
TLR-4 & TGGATACGTTTCCTTATAAG & GAAATGGAGGCACCCCTTC \\
IL-1 $\beta$ & TGATGGCTTATTACAGTGGCAATG & GTAGTGGTGGTCGGAGATTCG \\
IL-10 & ACCTGCCTAACATGCTTCGAG & CTGGGTCTTGGTTCTCAGCTT \\
TNF- $\alpha$ & TGCTTGTTCCTCAGCCTCTT & CAGAGGGCTGATTAGAGAGAGGT \\
GAPDH & ACAGTCAGCCGCATCTTCTT & ACGACCAAATCCGTTGACTC
\end{tabular}

Dectin-1, dendritic cell-associated C-type lectin receptor-1; TLR, toll-like receptor; IL, interleukin; TNF, tumor necrosis factor.

\section{Results}

Overexpression and silencing of PU.1 in THP-1-derived macrophages. A recombinant adenoviral vector carrying the PU.1 gene SPII (Ad-PU.1) was provided by Shanghai R\&S Biotechnology Co., Ltd. and used to transduce THP-1 derived macrophages. RT-qPCR analysis demonstrated that PU.1 mRNA increased by 80.4 -fold in the Ad-PU.1 group when compared with the Mock group ( $\mathrm{P}<0.05$; Fig. 1A). Western blot analysis confirmed the results of the RT-qPCR analysis (Fig. 1B). In addition, RT-qPCR analysis demonstrated that PU.1 mRNA levels were reduced to $19.0 \%$ of the Mock group following transfection with siPU.1 ( $\mathrm{P}<0.05$; Fig. 1C). Consistent with these observations, western blotting revealed that PU.1 protein levels were markedly decreased in the siPU.1-transfected group when compared with the mock group (Fig. 1D). The results demonstrated that PU.1 was successfully overexpressed and silenced in THP-1-derived macrophages.

Expression of Dectin-1, TLR-2 and TLR-4 following overexpression and silencing of PU.1. As PU.1 is essential for the transcriptional regulation of Dectin-1, TLR-2 and TLR-4 (13-18), the mRNA and protein expression levels of these genes in THP-1-derived macrophages following transduction with Ad-PU.1 and transfection with siPU.1 were analyzed. When compared with the mock group, Dectin-1, TLR-2 and TLR-4 mRNA expression increased by 8.31, 2.56 and 4.28-fold, respectively (Fig. 2A). Similarly, western blot analysis revealed that the protein expression levels of these three receptors increased when compared with the mock group (Fig. 2B). Conversely, the siPU.1 group exhibited a significant reduction in Dectin-1, TLR-2 and TLR-4 mRNA expression levels by 4.64, 4.04 and 4.33-fold, respectively, when compared with the mock controls (Fig. 2C). Consistent with these results, the protein expression levels of these factors were decreased in the siPU.1 group when compared with the mock group (Fig. 2D). The results indicate that PU.1 overexpression led to upregulation of Dectin-1, TLR-2 and TLR-4, and silencing of PU.1 led to downregulation of Dectin-1, TLR-2 and TLR-4.

Cytokine release, phagocytosis and the killing ability of THP-1-derived macrophages following overexpression of PU.1. In order to investigate whether overexpression of PU.1 promotes immune defense against $A$. fumigatus, the cytokine release, phagocytosis and killing ability of THP-1-derived macrophages was examined. Cells were stimulated for 0,4 , 8 and $12 \mathrm{~h}$ with resting conidia $(\mathrm{MOI}=1)$ at $48 \mathrm{~h}$ following transduction with Ad-PU.1. The mRNA levels of TNF- $\alpha$ and IL-1 $\beta$ increased in a time-dependent manner in the Ad-PU.1 group, whereas, IL-10 exhibited no significant increase in expression (Fig. 3A-C). The protein expression patterns of TNF- $\alpha$ and IL-10 in cell culture supernatants were consistent with the observed mRNA expression patterns (Fig. 3D and E).

AMs constitute the first line of a host's immune defense, and facilitate the initiation of the innate immune response to A. fumigatus infections, including phagocytosis and killing $(23,24)$. Therefore, the phagocytosis and killing ability of THP-1-derived macrophages following overexpression of PU.1 was examined. Cells were stimulated with resting conidia (MOI=0.5) for 4 and $8 \mathrm{~h}$, respectively. The killing rate in the Ad-PU.1 group was significantly higher when compared with the control groups (Fig. 3F). For the analysis of phagocytosis, cells were stimulated with FITC-labeled resting conidia (MOI=5) for $4 \mathrm{~h}$. The confocal laser-scanning microscopy results shown in Fig. 3G, demonstrated that phagocytosis was enhanced following overexpression of PU.1. The results therefore suggest that overexpression of PU.1 promoted cytokine release, and enhanced phagocytosis and the killing ability of THP-1-derived macrophages during A. fumigatus conidia stimulation.

Cytokine release, phagocytosis and the killing ability of THP-1-derived macrophages following silencing of PU.1. As overexpression of PU.1 appears to enhance the immune defense against $A$. fumigatus, the authors of the present study investigated whether silencing of PU.1 expression was associated with decreased cytokine release, phagocytosis and a reduced killing ability of THP-1-derived macrophages. Cells were stimulated for $0,4,8$ and $12 \mathrm{~h}$ with resting conidia (MOI=1) at $48 \mathrm{~h}$ following transfection with PU.1 siRNA. The mRNA levels of TNF- $\alpha$ and IL- $1 \beta$ decreased, while IL-10 levels increased significantly in the siPU-1 group when compared with the mock group (Fig. 4A-C). Similar results were observed for the protein levels of TNF- $\alpha$ and IL-10 in culture supernatants (Fig. 4D and E). To determine the killing ability, cells were stimulated with resting conidia (MOI=0.5) 
A

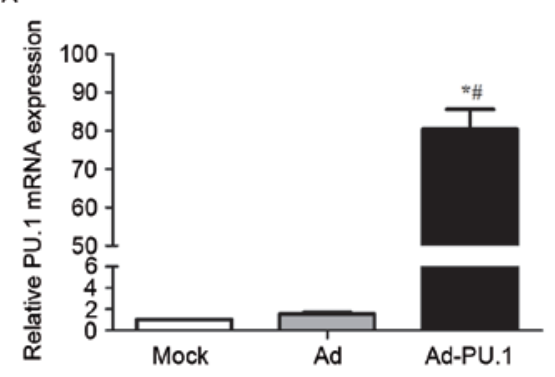

B

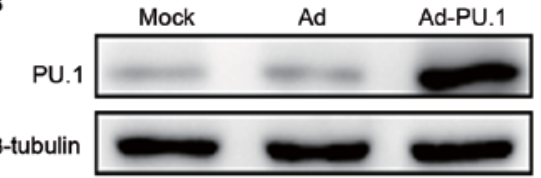

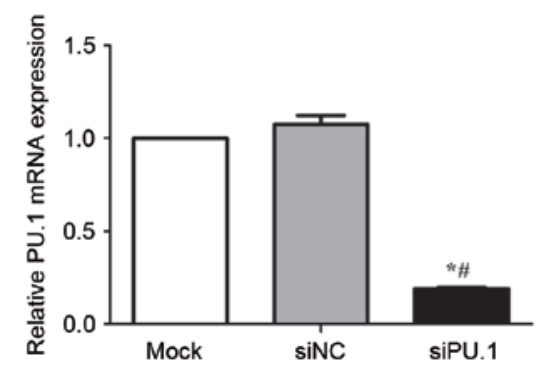

D

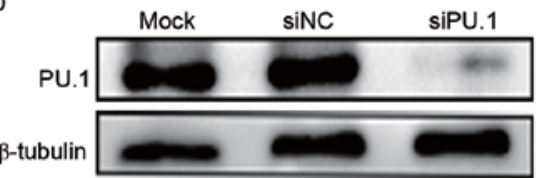

Figure 1. Relative PU.1 (A) mRNA and (B) protein expression levels were measured by RT-qPCR and western blot analyses, respectively, in mock, Ad and Ad-PU.1-transduced THP-1 cells ( $\mathrm{P}<0.05$ vs. Mock group; ${ }^{*} \mathrm{P}<0.05$ vs. Ad group). Relative PU.1 (C) mRNA and (D) protein expression levels were measured by RT-qPCR and western blotting analyses, respectively, in mock, siNC and siPU.1-transfected THP-1 cells ( ${ }^{*} \mathrm{P}<0.05$ vs. Mock group; ${ }^{\#} \mathrm{P}<0.05$ vs. siNC group). RT-qPCR, reverse transcription-quantitative polymerase chain reaction; Ad-PU.1, recombinant adenoviral-PU.1 vector; siPU.1, small-interfering RNA targeting PU.1; mock, untransfected/transduced cells; Ad, adenovirus-transduced cells; siNC, negative control siRNA.

A

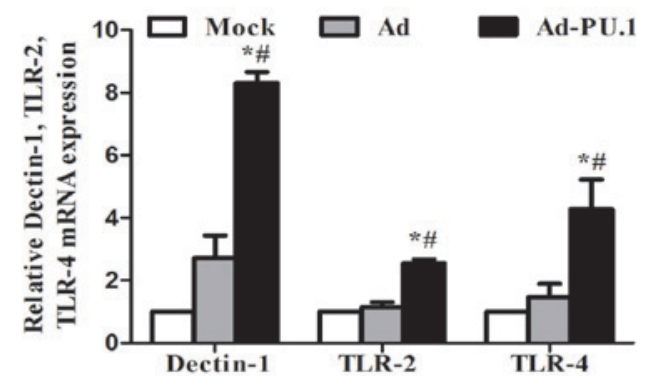

B

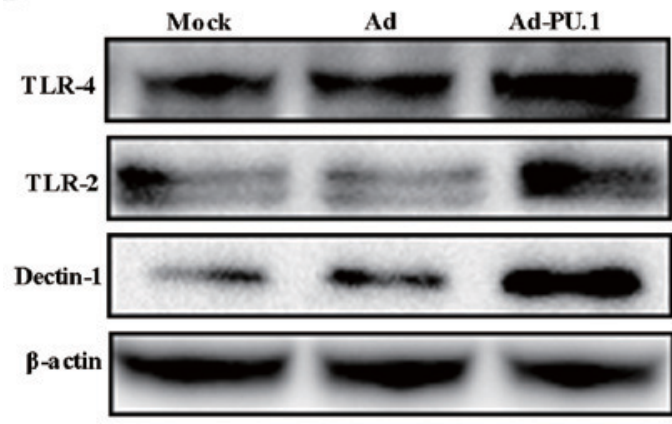

C

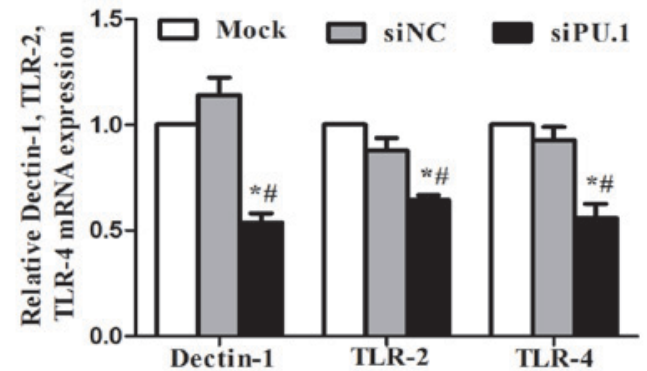

D

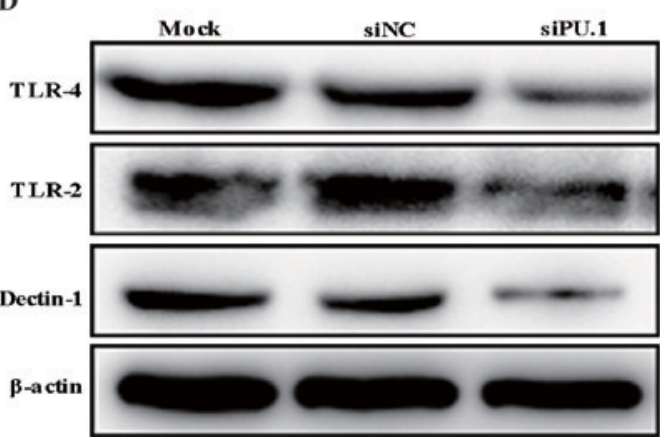

Figure 2. Relative levels of Dectin-1, TLR-2 and TLR-4 (A) mRNA and (B) protein expression in THP-1 cells at 48 h following transduction with AdPU.1, as determined by RT-qPCR and western blot analyses, respectively ( $\mathrm{P}<0.05$ vs. Mock group; ${ }^{*} \mathrm{P}<0.05$ vs. Ad group). Relative levels of Dectin-1, TLR-2 and TLR-4 (C) mRNA and (D) protein expression in THP-1 cells at $48 \mathrm{~h}$ following transfection with siPU.1, as determined by RT-qPCR and western blot analyses, respectively ("P<0.05 vs. Mock group; ${ }^{*} \mathrm{P}<0.05$ vs. siNC group). Dectin-1, dendritic cell-associated C-type lectin receptor-1; TLR, toll-like receptor; RT-qPCR, reverse transcription-quantitative polymerase chain reaction; Ad-PU.1, recombinant adenoviral-PU.1 vector; siPU.1, small-interfering RNA targeting PU.1; mock, untransfected/transduced cells; Ad, adenovirus-transduced cells; siNC, negative control siRNA.

for 4 and 8 h. As shown in Fig. 4F, the killing rate in siPU.1 group was significantly lower compared with the control groups at $4 \mathrm{~h}$; however, no significant difference at $8 \mathrm{~h}$ was observed (Fig. 4F). Following the stimulation of cells with FITC-labeled resting conidia (MOI=5) for $4 \mathrm{~h}$, a decrease in phagocytosis was observed in the siPU.1 group when compared with the controls (Fig. 4G). These results demonstrate that silencing of PU.1 decreased cytokine release, phagocytosis and the killing ability of THP-1-derived macrophages during A. fumigatus conidia stimulation.

\section{Discussion}

A. fumigatus resting conidia are inhaled frequently and, if not effectively cleared by the innate immune system, the conidia germinate and form invasive hyphae, which activate innate and 
A

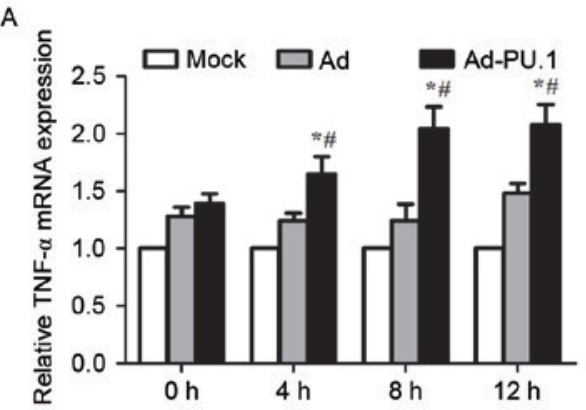

B

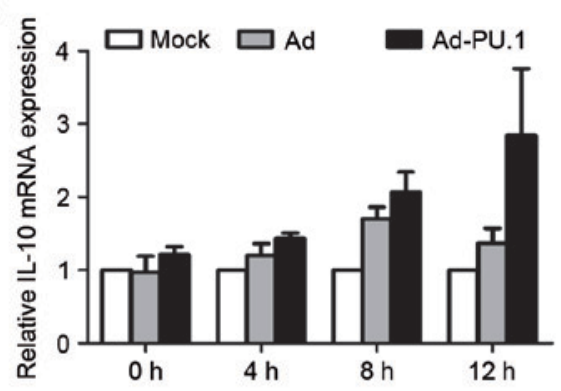

C

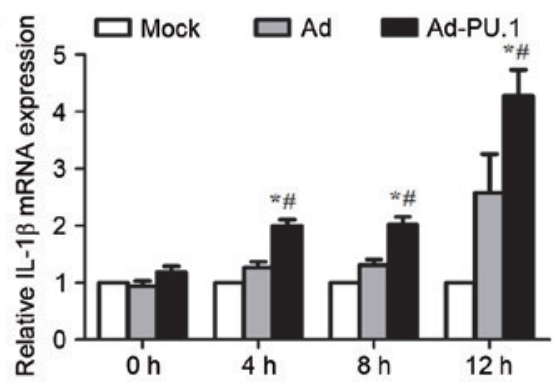

D

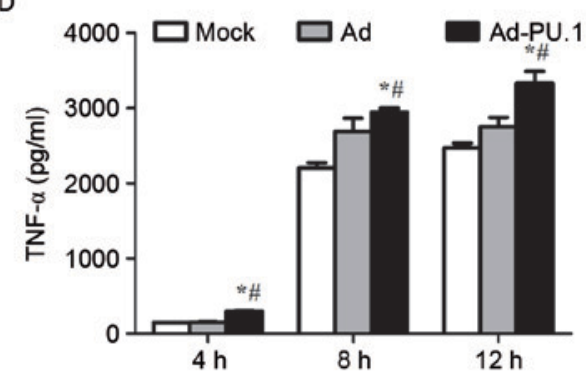

E

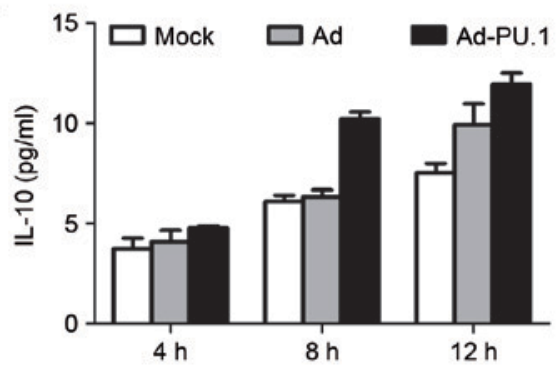

F

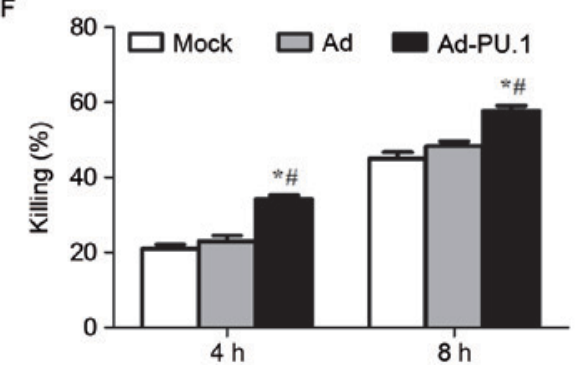

G

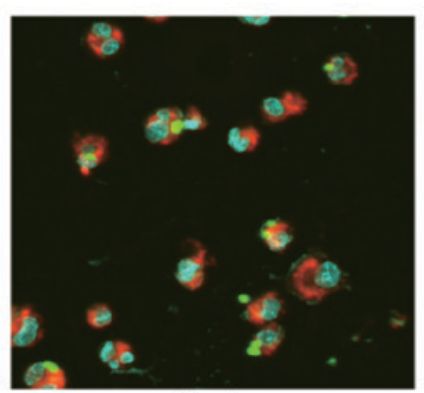

Mock

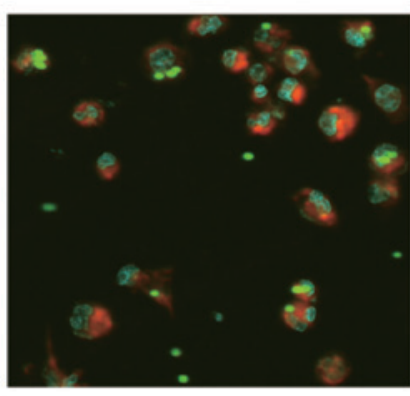

Ad

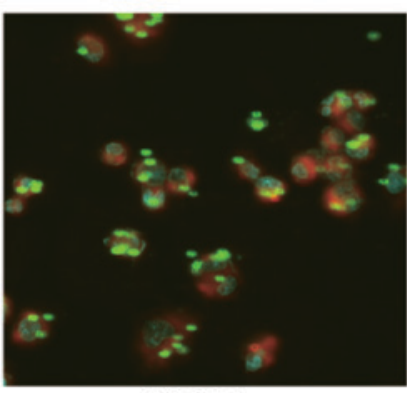

Ad-PU.1

Figure 3. mRNA levels of (A) TNF- $\alpha$, (B) IL-10 and (C) IL-1 $\beta$ in mock, Ad, or Ad-PU.1-transduced THP-1 cells at 0, 4, 8 and $12 \mathrm{~h}$ of exposure to resting conidia, as determined by reverse transcription-quantitative polymerase chain reaction analysis. The protein levels of (D) TNF- $\alpha$ and (E) IL-10 were assayed by enzyme-linked immunosorbent assay analysis. (F) Killing ability was assessed by serial dilution of cells following co-incubation with resting conidia, and quantification of the number of colony forming units. (G) Confocal laser-scanning microscopy was used to observe phagocytosis after $4 \mathrm{~h}$ of incubation with resting conidia. Magnification, $\mathrm{x} 63$ (objective oil immersion lens). " $\mathrm{P}<0.05$ vs. Mock group; ${ }^{*} \mathrm{P}<0.05 \mathrm{vs}$. Ad group. TNF, tumor necrosis factor; IL, interleukin; Mock, untransduced cells; Ad, adenovirus-transduced cells; Ad-PU.1, recombinant adenoviral-PU.1 vector.

adaptive immune responses (25). The innate immune response is mediated by phagocytic cells, primarily macrophages, which prevent the growth of inhaled conidia and hyphae. Recognition of conidia by different PRRs expressed on the membrane of AMs leads to the phagocytosis and killing of inhaled conidia, and induces the expression of inflammatory cytokines and chemokines (23).

PU.1 is an important transcription factor that belongs to the Ets family, and is a critical regulator of cellular communication in the immune system (26). AMs are the only mature tissue macrophages that express PU.1 (27). PU.1 regulates several genes that serve critical roles in cellular functions, including phagocytosis, TLR signaling pathways, CTLs and surfactant clearance $(14,15,28)$. Overexpression of PU.1 using a retrovirus vector has been reported in two important studies $(15,27)$. One such study demonstrated that retrovirus-mediated expression of PU.1 in AMs from $\mathrm{GM}^{-1-}$ mice rescued the marked reduction in TLR-2 and TLR-4 mRNA expression (15). The other study demonstrated that overexpression of PU.1 in bone marrow-derived dendritic cells induced the expression of cluster of differentiation (CD) 80 and CD86, suggesting that PU.1 may be a potential target for the treatment of immune-associated diseases (29). Similar to the results of previous studies $(14,16,17)$, the present study 
A

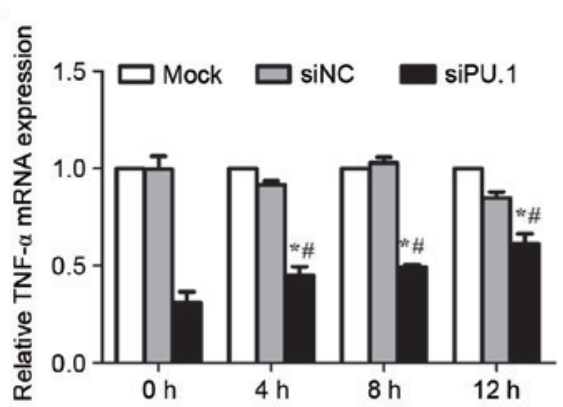

B

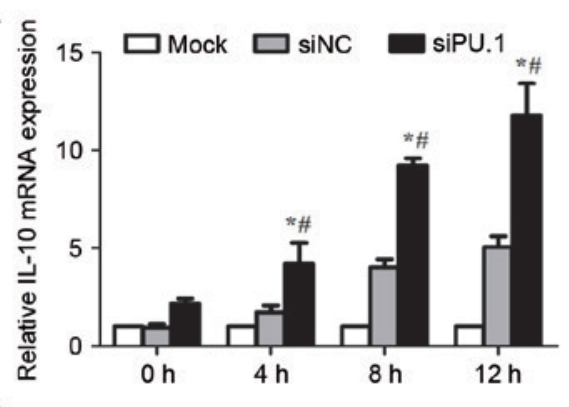

C

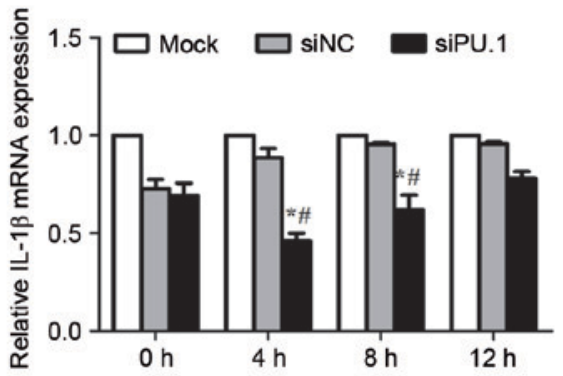

D

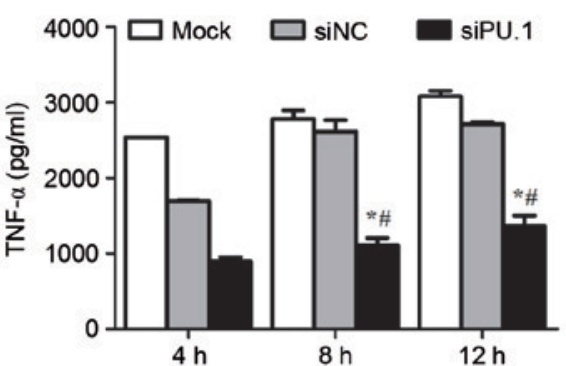

E
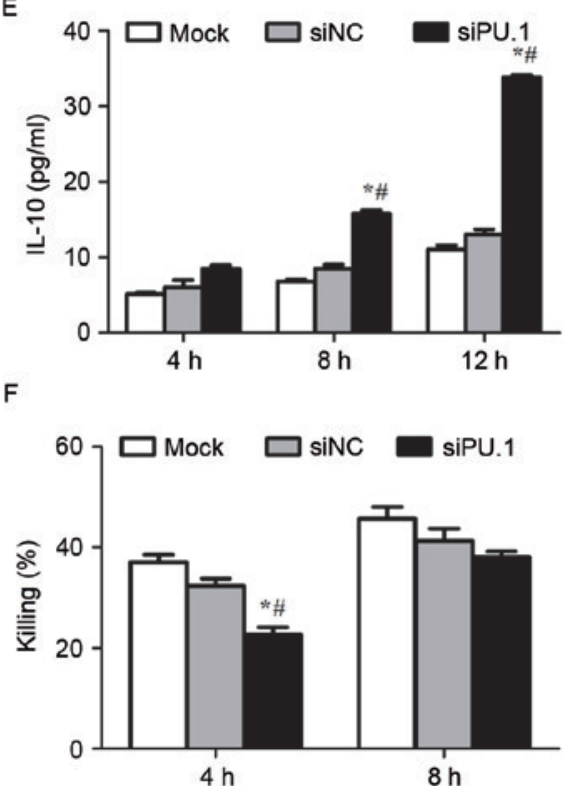

G

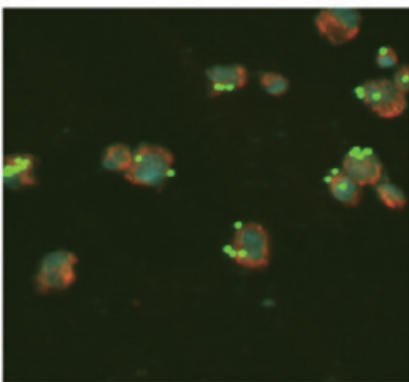

Mock

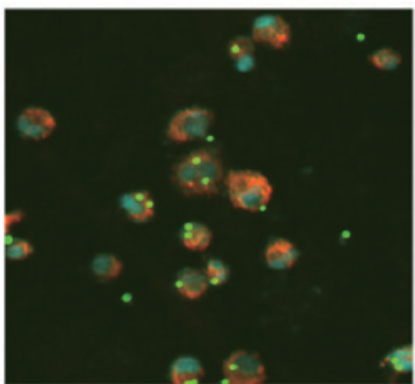

siNC

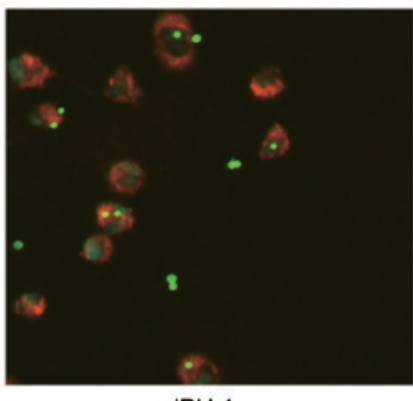

siPU.1

Figure 4. mRNA levels of (A) TNF- $\alpha$, (B) IL-10 and (C) IL-1 $\beta$ in mock, siNC, or siPU.1-transfected THP-1 cells at $0,4,8$ and $12 \mathrm{~h}$ of exposure to resting conidia, as determined by reverse transcription-quantitative polymerase chain reaction analysis. The protein levels of (D) TNF- $\alpha$ and (E) IL-10 were assayed by enzyme-linked immunosorbent assay analysis. (F) Killing ability was assessed by serial dilution of cells following co-incubation with resting conidia, and quantification of the number of colony forming units. $(\mathrm{G})$ Confocal laser-scanning microscopy was used to observe phagocytosis after $4 \mathrm{~h}$ of incubation with resting conidia. Magnification, $\mathrm{x} 63$ (objective oil immersion lens). ${ }^{\mathrm{P}}<0.05$ vs. Mock group; ${ }^{\text {}} \mathrm{P}<0.05$ vs. siNC group. TNF, tumor necrosis factor; IL, interleukin; Mock, untransfected cells; siNC, negative control small-interfering RNA; siPU.1, small-interfering RNA targeting PU.1.

observed decreased expression levels of TLR-2, TLR-4 and Dectin-1 following silencing of PU.1 expression. However, an adenoviral vector containing the PU.1 gene was successfully constructed in the present study, and the results revealed that overexpression of PU.1 leads to the increase of Dectin-1,TLR-2 and TLR-4 expression at the transcriptional and translational levels. Although its precise regulatory mechanism has not yet been fully elucidated, the results from gene overexpression and silencing experiments have demonstrated that PU.1 may be an essential regulator of Dectin-1, TLR-2 and TLR-4 expression in THP-1-derived macrophages.
Following inhalation, A. fumigatus conidia undergo a progression from conidial swelling to hyphal growth. During this process, conidia cell wall components are exposed and are detected by various PRRs on macrophages, particularly CTLs and TLRs (30). Dectin-1, TLR-2 and TLR-4 are the major PRRs on phagocytic cells that recognize fungal components (31). In human and murine macrophages, several studies have demonstrated that Dectin-1, TLR-2 and TLR-4 are involved in the secretion of pro-inflammatory cytokines in response to live and dead A. fumigatus $(3,20,32-34)$. TNF- $\alpha$ and IL-1 $\beta$ are major pro-inflammatory cytokines, and IL-10 is 
a key anti-inflammatory cytokine. Notably, TNF- $\alpha$ enhances the host response to A. fumigatus during the early and later stages of infection (4). In the present study, THP-1-derived macrophages were stimulated with A.fumigatus resting conidia following overexpression or silencing of PU.1 expression. TNF- $\alpha$ and IL-1 $\beta$ levels increased significantly and in a time-dependent manner in THP-1 cells following overexpression of PU.1 and exposure to A. fumigatus, whereas IL-10 exhibited no significant increase. By contrast, TNF- $\alpha$ and IL-1 $\beta$ levels decreased significantly following PU.1 silencing. The present study demonstrated that overexpression of PU.1 increased Dectin-1, TLR-2 and TLR-4 expression, which led to increased cytokine levels during A. fumigatus stimulation. As expected, silencing of PU.1 led to a decrease in the levels of these receptors and cytokines.

Dectin-1, TLR-2 and TLR-4 facilitate the recognition of several morphological forms of A. fumigatus, including swollen conidia, germinating conidia and hyphae (not resting conidia) (4). A. fumigatus conidia begin to swell at $\sim 3 \mathrm{~h}$ following inhalation, and swollen conidia are prerequisites for efficient phagocytosis and killing $(24,35)$. Therefore, the ability of THP-1-derived macrophages to phagocytose resting conidia following $4 \mathrm{~h}$ of co-incubation was examined using confocal laser-scanning microscopy in the present study. The results demonstrated that phagocytosis was markedly enhanced in the Ad-PU.1 group when compared with the control groups. By contrast, a reduction in phagocytosis was observed in the siPU.1 group when compared with the control group. Killing is commonly induced following conidia phagocytosis (36). The killing ability of THP-1-derived macrophages following overexpression or silencing of PU.1 expression was examined further, and the results demonstrated that the killing ability of the THP-1-derived macrophages was significantly higher in the Ad-PU.1 group when compared with the control groups. In the siPU.1 group, the killing ability following conidia stimulation was lower following PU.1 silencing when compared with the control groups.

Previous studies have demonstrated that PU.1 induced the expression of a number of genes that have critical roles in the immune response to several infectious pathogens, including Mycobacterium tuberculosis, pneumocystis, Epstein-Barr virus and Ehrlichia chaffeensis (14,37-39). In a previous in vivo study (40), decreased inflammatory cytokine release and survival benefits were observed in mice with macrophages that lacked functional PU.1, which were challenged with lipopolysaccharide, a component of the outer membrane of gram-negative bacteria. Thus, PU.1 serves a protective mechanistic role in response to endotoxemia. To the best of our knowledge, the present study was the first to demonstrate that PU.1 serves a role in A.fumigatus infection. The results demonstrated that PU.1 may function to induce pro-inflammatory cytokines, such as TNF- $\alpha$ and IL- $1 \beta$, and the anti-inflammatory cytokine IL-10. Of particular note, excessive inflammation may negatively affect the host; therefore, efficient protection against fungal infection requires a fine-tuned balance between pro-inflammatory cytokines and anti-inflammatory cytokines.

In conclusion, the present study constructed a recombinant adenoviral vector containing the PU.1 gene and generated an AM model that overexpressed and silenced PU.1 expression.
The results demonstrated that Dectin-1, TLR-2 and TLR-4 expression were upregulated and downregulated following overexpression and silencing of PU.1, respectively. It was confirmed that overexpression of PU.1 promoted the release of pro-inflammatory cytokines, and enhanced the phagocytosis and killing ability of THP-1-derived macrophages during A. fumigatus stimulation. Indeed, silencing of PU.1 led to a reduction in the release of pro-inflammatory cytokines, phagocytosis, and the killing ability of THP-1-derived macrophages. Therefore, PU.1 may be an essential factor for defense against A. fumigatus infection, and overexpression of PU.1 may be a novel method for the prophylaxis or treatment of IPA.

\section{Acknowledgements}

The present study was supported by grants from the National Natural Science Foundation of China (grant nos. 81270064, 81200063 and 81200004).

\section{References}

1. Sugui JA, Kwon-Chung KJ, Juvvadi PR, Latge JP and Steinbach WJ: Aspergillus fumigatus and Related Species. Cold Spring Harb Perspect Med 5: a19786, 2015.

2. Cramer RA, Rivera A and Hohl TM: Immune responses against Aspergillus fumigatus: What have we learned? Curr Opin Infect Dis 24: 315-322, 2011.

3. Xia D, Sun WK, Tan MM, Ding Y, Liu ZC, Li P, Qian Q, Su X and Shi Y: An adenoviral vector encoding full-length dectin-1 promotes aspergillus-induced innate immune response in macrophages. Lung 193: 549-557, 2015.

4. Margalit A and Kavanagh K: The innate immune response to Aspergillus fumigatus at the alveolar surface. Fems Microbiol Rev 39: 670-687, 2015.

5. Werner JL, Metz AE, Horn D, Schoeb TR, Hewitt MM, Schwiebert LM, Faro-Trindade I, Brown GD and Steele C: Requisite role for the dectin-1 beta-glucan receptor in pulmonary defense against Aspergillus fumigatus. J Immunol 182: 4938-4946, 2009.

6. Cunha C, Di Ianni M, Bozza S, Giovannini G, Zagarella S, Zelante T, D'Angelo C, Pierini A, Pitzurra L, Falzetti F, et al: Dectin-1 Y238X polymorphism associates with susceptibility to invasive aspergillosis in hematopoietic transplantation through impairment of both recipient- and donor-dependent mechanisms of antifungal immunity. Blood 116: 5394-5402, 2010.

7. Hohl TM and Feldmesser M: Aspergillus fumigatus: Principles of pathogenesis and host defense. Eukaryot Cell 6: 1953-1963, 2007.

8. Mehrad B, Strieter RM and Standiford TJ: Role of TNF-alpha in pulmonary host defense in murine invasive aspergillosis. J Immunol 162: 1633-1640, 1999.

9. Meier A, Kirschning CJ, Nikolaus T, Wagner H, Heesemann J and Ebel F: Toll-like receptor (TLR) 2 and TLR4 are essential for aspergillus-induced activation of murine macrophages. Cell Microbiol 5: 561-570, 2003.

10. Bellocchio S, Montagnoli C, Bozza S, Gaziano R, Rossi G, Mambula SS, Vecchi A, Mantovani A, Levitz SM and Romani L: The contribution of the toll-like/IL-1 Receptor superfamily to innate and adaptive immunity to fungal pathogens in vivo. J Immunol 172: 3059-3069, 2004.

11. Bochud PY, Chien JW, Marr KA, Leisenring WM, Upton A, Janer M, Rodrigues SD, Li S, Hansen JA, Zhao LP, et al: Toll-like receptor 4 polymorphisms and aspergillosis in stem-cell transplantation. N Engl J Med 359: 1766-1777, 2008.

12. Ghisletti S, Barozzi I, Mietton F, Polletti S, De Santa F, Venturini E, Gregory L, Lonie L, Chew A, Wei CL, et al: Identification and characterization of enhancers controlling the inflammatory gene expression program in macrophages. Immunity 32: 317-328, 2010.

13. Serezani CH, Kane S, Collins L, Morato-Marques M, Osterholzer JJ and Peters-Golden M: Macrophage dectin-1 expression is controlled by leukotriene B4 Via a GM-CSF/PU.1 axis. J Immunol 189: 906-915, 2012. 
14. Zhang C, Wang SH, Liao CP, Shao S, Lasbury ME, Durant PJ and Lee CH: Downregulation of PU.1 leads to decreased expression of dectin-1 in alveolar macrophages during pneumocystis pneumonia. Infect Immun 78: 1058-1065, 2010.

15. Shibata Y, Berclaz PY, Chroneos ZC, Yoshida M, Whitsett JA and Trapnell BC: GM-CSF regulates alveolar macrophage differentiation and innate immunity in the lung through PU.1. Immunity 15: 557-567, 2001.

16. Haehnel V, Schwarzfischer L, Fenton MJ and Rehli M: Transcriptional regulation of the human toll-like receptor 2 gene in monocytes and macrophages. J Immunol 168: 5629-5637, 2002.

17. Park SY, Lee SW, Baek SH, Lee CW, Lee WS, Rhim BY, Hong KW and Kim CD: Suppression of PU.1-linked TLR4 expression by cilostazol with decrease of cytokine production in macrophages from patients with rheumatoid arthritis. Br J Pharmacol 168: 1401-1411, 2013.

18. Moon HG, Yang J, Zheng Y and Jin Y: MiR-15a/16 regulates macrophage phagocytosis after bacterial infection J Immunol 193: 4558-4567, 2014.

19. Persat F, Noirey N, Diana J, Gariazzo MJ, Schmitt D, Picot S and Vincent C: Binding of live conidia of Aspergillus fumigatus activates in vitro-generated human langerhans cells via a lectin of galactomannan specificity. Clin Exp Immunol 133: 370-377, 2003.

20. Sun WK, Lu X, Li X, Sun QY, Su X, Song Y, Sun HM and Shi Y: Dectin-1 is inducible and plays a crucial role in Aspergillus-induced innate immune responses in human bronchial epithelial cells. Eur J Clin Microbiol Infect Dis 31: 2755-2764, 2012.

21. Livak KJ and Schmittgen TD: Analysis of relative gene expression data using real-time quantitative PCR and the 2(-Delta Delta C(T)) method. Methods 25: 402-408, 2001.

22. Sun H, Xu XY, Tian XL, Shao HT, Wu XD, Wang Q, Su X and Shi $\mathrm{Y}$ : Activation of $\mathrm{NF}-\kappa \mathrm{B}$ and respiratory burst following Aspergillus fumigatus stimulation of macrophages. Immunobiology 219: 25-36, 2014.

23. Heinekamp T, Schmidt H, Lapp K, Pähtz V, Shopova I, Köster-Eiserfunke N, Krüger T, Kniemeyer O and Brakhage AA Interference of Aspergillus fumigatus with the immune response. Semin Immunopathol 37: 141-152, 2015.

24. Ibrahim-Granet O, Philippe B, Boleti H, Boisvieux-Ulrich E, Grenet D, Stern M and Latgé JP: Phagocytosis and intracellular fate of Aspergillus fumigatus conidia in alveolar macrophages. Infect Immun 71: 891-903, 2003.

25. Dagenais TR and Keller NP: Pathogenesis of Aspergillus fumigatus in invasive aspergillosis. Clin Microbiol Rev 22: 447-465, 2009

26. Turkistany SA and DeKoter RP: The transcription factor PU.1 is a critical regulator of cellular communication in the immune system. Arch Immunol Ther Exp (Warsz) 59: 431-440, 2011.

27. Nakata K, Kanazawa $\mathrm{H}$ and Watanabe M: Why does the autoantibody against granulocyte-macrophage colony-stimulating factor cause lesions only in the lung? Respirology 11 (Suppl): S65-S69, 2006.
28. Trapnell BC and Whitsett JA: Gm-CSF regulates pulmonary surfactant homeostasis and alveolar macrophage-mediated innate host defense. Annu Rev Physiol 64: 775-802, 2002.

29. Kanada S, Nishiyama C, Nakano N, Suzuki R, Maeda K, Hara M, Kitamura N, Ogawa $\mathrm{H}$ and Okumura K: Critical role of transcription factor PU.1 in the expression of CD80 and CD86 on dendritic cells. Blood 117: 2211-2222, 2011.

30. Becker KL, Ifrim DC, Quintin J,Netea MG and van de Veerdonk FL: Antifungal innate immunity: Recognition and inflammatory networks. Semin Immunopathol 37: 107-116, 2015.

31. Bonfim CV, Mamoni RL and Blotta MH: TLR-2, TLR-4 and Dectin-1 expression in human monocytes and neutrophils stimulated by paracoccidioides brasiliensis. Med Mycol 47: 722-733, 2009.

32. Braedel S, Radsak M, Einsele H, Latgé JP, Michan A, Loeffler J, Haddad Z, Grigoleit U, Schild H and Hebart H: Aspergillus fumigatus antigens activate innate immune cells via toll-like receptors 2 and 4. Br J Haematol 125: 392-399, 2004.

33. Mambula SS, Sau K, Henneke P, Golenbock DT and Levitz SM: Toll-like receptor (TLR) signaling in response to Aspergillus fumigatus. J Biol Chem 277: 39320-39326, 2002.

34. Meier A, Kirschning CJ, Nikolaus T, Wagner H, Heesemann J and Ebel F: Toll-like receptor (TLR) 2 and TLR4 are essential for Aspergillus-induced activation of murine macrophages. Cell Microbiol 5: 561-570, 2003

35. Marr KA, Balajee SA, Hawn TR, Ozinsky A, Pham U, Akira S, Aderem A and Liles WC: Differential role of MyD88 in macrophage-mediated responses to opportunistic fungal pathogens. Infect Immun 71: 5280-5286, 2003.

36. Philippe B, Ibrahim-Granet O, Prévost MC, GougerotPocidalo MA, Sanchez PM, Van der Meeren A and Latgé JP: Killing of Aspergillus fumigatus by alveolar macrophages is mediated by reactive oxidant intermediates. Infect Immun 71: 3034-3042, 2003.

37. Zhang G, Zhou B, Li S, Yue J, Yang H, Wen Y, Zhan S, Wang W, Liao M, Zhang M, et al: Allele-specific induction of IL-1 $\beta$ expression by $\mathrm{C} / \mathrm{EBP} \beta$ and PU.1 contributes to increased tuberculosis susceptibility. Plos Pathog 10: e1004426, 2014.

38. Lin JH, Lin JY, Chou YC, Chen MR, Yeh TH, Lin CW, Lin SJ and Tsai CH: Epstein-barr virus LMP2A suppresses MHC class II expression by regulating the b-cell transcription factors E47 and PU.1. Blood 125: 2228-2238, 2015.

39. Lin M and Rikihisa Y: Ehrlichia chaffeensis downregulates surface toll-like receptors $2 / 4, \mathrm{CD} 14$ and transcription factors PU.1 and inhibits lipopolysaccharide activation of NF-kappa B, ERK 1/2 and P38 MAPK in host monocytes. Cell Microbiol 6: $175-186,2004$

40. Karpurapu M, Wang X, Deng J, Park H, Xiao L, Sadikot RT, Frey RS, Maus UA, Park GY, Scott EW and Christman JW: Functional PU.1 in macrophages has a pivotal role in NF- $\kappa$ B activation and neutrophilic lung inflammation during endotoxemia. Blood 118: 5255-5266, 2011. 